\title{
Operando and multimodal studies of speciation and activity of Pt catalysts during the hydrogenation of ethylene
}

Eric A. Stach, ${ }^{1}$ Shen Zhao, ${ }^{1,2}$ Yuanyuan Li, ${ }^{3}$ Deyu Liu, ${ }^{2}$ Jing Liu, ${ }^{3}$ Yao-Min Liu, ${ }^{2}$ Dmitri N. Zakharov, ${ }^{1}$ Qiyuan Wu, ${ }^{3}$ Alexander Orlov, ${ }^{3}$ Andrew A. Gewirth, ${ }^{2}$ Ralph G. Nuzzo, ${ }^{3}$ Anatoly I. Frenkel ${ }^{3,4}$

${ }^{1}$ Center for Functional Nanomaterials, Brookhaven National Laboratory, Upton, NY 11793

${ }^{2}$ Department of Chemistry, University of Illinois, Urbana, IL 61801

${ }^{3}$ Department of Materials Science and Chemical Engineering, Stony Brook University, Stony Brook, NY 11794

${ }^{4}$ Department of Chemistry, Brookhaven National Laboratory, Upton, NY 11793

The creation of fuels and large volume chemicals (such as olefins) from crude oil feedstocks involves the hydrogenation of unsaturated hydrocarbons. These processes involve numerous catalytic reforming and hydrogenation/dehydrogenation processes, and are generally mediated by supported metal nanoparticle catalysts. These catalysts are generally chosen for their high activity, long term stability and the ease with which they can be regenerated and recovered. However, despite the extensive use of these materials, there are many questions that remain about how specific attributes of the structure and composition of the catalysts are affected by the gases with which they interact. Furthermore, it is critically important to understand how these structural changes affect selectivity, as well as how deactivation occurs because of the conversion process.

In this work, we will describe how we use a multi-modal, operando experimental approach to understand the subtle changes that occur to both the atomic structure and the chemical state of palladium nanoparticle catalysts supported on $\mathrm{SiO}_{2}$ during the hydrogenation of ethylene. The core of this approach is the use of a closed-cell microreactor [1] that allows sequential experimental investigation via scanning transmission electron microscopy (STEM), x-ray absorption spectroscopy (XAS) and microbeam infrared spectroscopy ( $\mu$-IR), and gas-chromotography/mass spectroscopy (GC-MS), with all measurements being made in the same operando reaction conditions.[2]

We will describe how this approach allows us to directly correlate the measurements in a robust fashion, leading to novel insights regarding several aspects of ethylene conversion. In specific, we will describe how the specifics of reactive gas composition lead to interconversion of both hydride and carbide phases of the Pd clusters, which processes affect the stability of the particles against coarsening, the reversibility of structural and compositional transformations and the role that surface oligomers that form under hydrogen limited reactant conditions, leading to deactivation.

[1] Li, Y., D. Zakharov, S. Zhao, R. Tappero, U. Jung, A. Elsen, Ph Baumann, Ralph G. Nuzzo, E. A. Stach, and A. I. Frenkel. Nature Comm. 6, 7583 (2015).

[2] Zhao, S., Li, Y., Stavitski, E., Tappero, R., Crowley, S., Castaldi, M.J., Zakharov, D.N., Nuzzo, R.G., Frenkel, A.I. and Stach, E.A., 2015. ChemCatChem, 7(22), pp.3683-3691.

[3] Zhao, S., Y. Li, D. Liu, J. Liu, Y.-M. Liu, D.N. Zakharov, Q. Wu, A. Orlov, A.A. Gewirth, E.A. Stach, R.G. Nuzzo, A.I. Frenkel, in review.

[4] The authors gratefully acknowledge support for this by the US Department of Energy, Office of Basic Energy Sciences under Grant No. DE-FG02-03ER15476. The development of the micro-cell was 
supported, in part, by an LDRD grant at Brookhaven National Laboratory. We acknowledge the facilities support provided at the Centre for Functional Nanomaterials, the National Synchrotron Light Source at the Brookhaven National Laboratory (US Department of Energy, Office of Basic Energy Sciences, Contract No. DE-SC0012704) and the Synchrotron Catalysis Consortium (US Department of Energy, Office of Basic Energy Sciences, Grant No. DE-SC0012335).

(a)
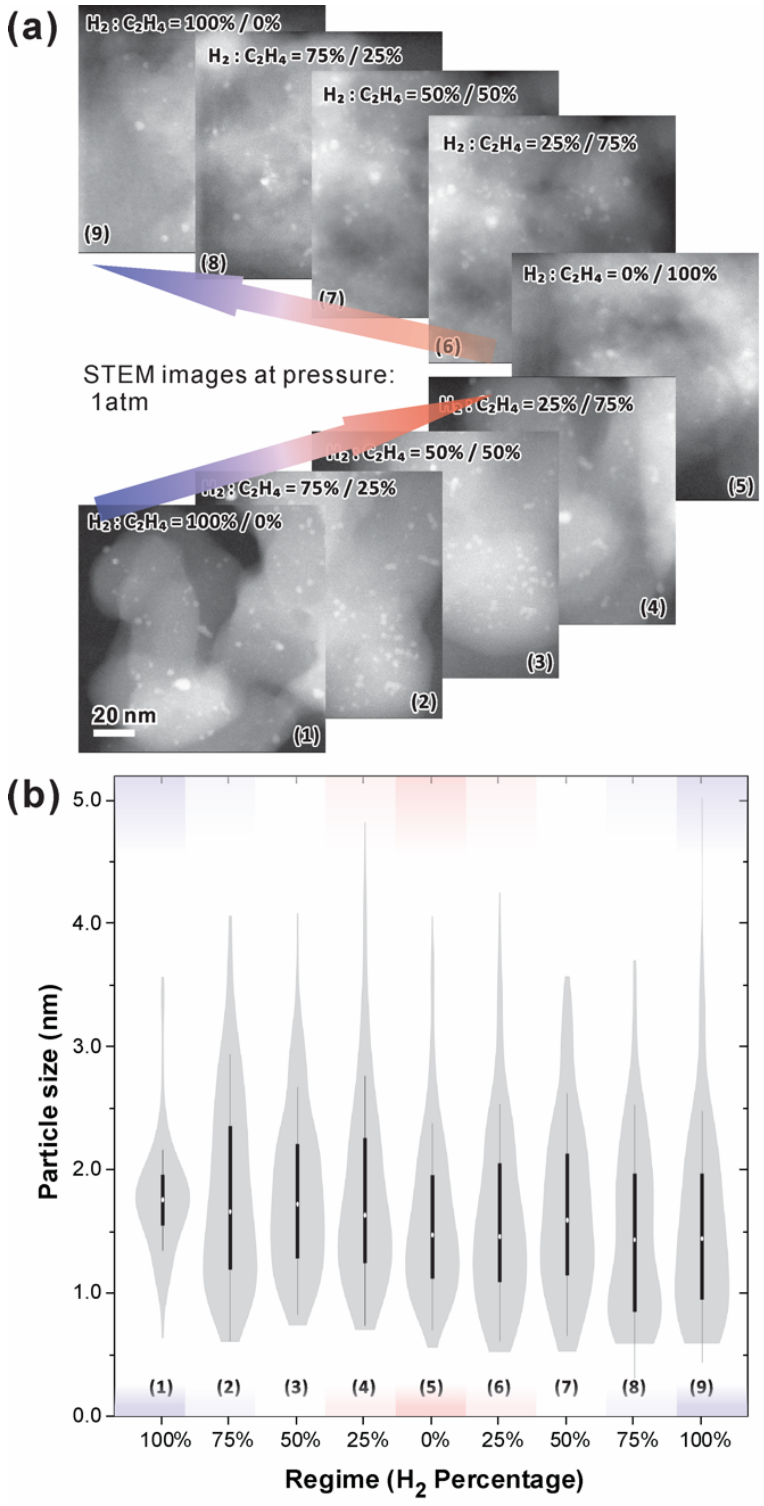

Figure 1. (a) Operando STEM images and (b) STEM measured particle size distributions of Pd NPs supported on $\mathrm{SiO}_{2}$ under different reaction stages at $1 \mathrm{~atm}$.
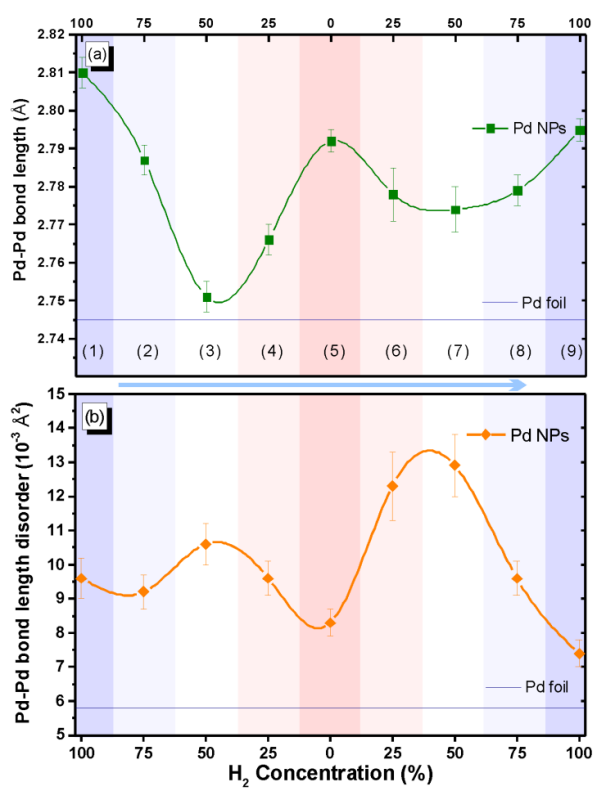

Figure 2: EXAFS analysis results of (a) the interatomic distances $r$ and (b) bond length disorder $\left(\sigma^{2}\right)$ for $\mathrm{Pd}-\mathrm{SiO}_{2}$ catalyst under different feed gas composition regimes of $\mathrm{H}_{2}$ and $\mathrm{C}_{2} \mathrm{H}_{4}$ (from pure $\mathrm{H}_{2}$ to pure $\mathrm{C}_{2} \mathrm{H}_{4}$ and then back to pure $\mathrm{H}_{2}$ ). The dashed line in (a) indicates the bond length of bulk Pd (foil).

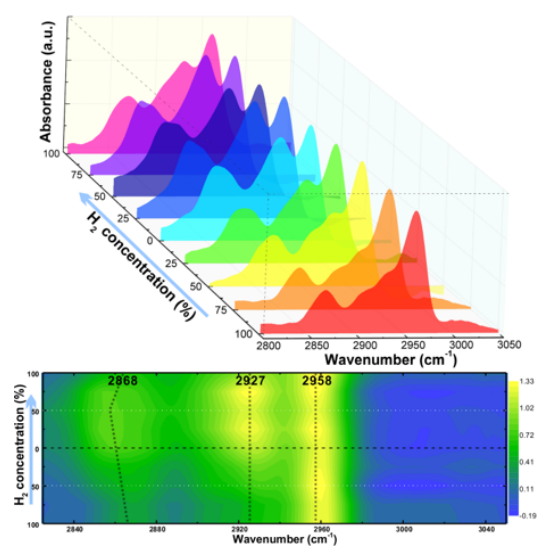

Figure 3: IR spectra collected in operando conditions under different regimes of feed gas concentration for room temperature ethylene hydrogenation over $\mathrm{Pd}-\mathrm{SiO}_{2}$ catalysts. Top waterfall figure show individual spectra acquired under different regimes, the lower contour map highlights the maxima of hydrocarbon fingerprint bands. 